(p. G367), ultrathin (<5 nm) $\mathrm{HfO}_{2}$ films were grown using atomic layer deposition on 12-in. $p$-type silicon (100) substrates. Xenon FLA was then conducted at capacitor charging voltages of 3100-3500 V with a $0.8-\mathrm{ms}$ pulse width. The samples were preheated to $400^{\circ} \mathrm{C}$ in a $\mathrm{N}_{2}$ atmosphere. $\mathrm{X}$ ray diffraction $(\mathrm{XRD})$ measurements were performed on the samples subjected to xenon FLA and conventional rapid thermal annealing. $\mathrm{TaSi}_{2.5}(10 \mathrm{~nm}) / \mathrm{W}(100 \mathrm{~nm})$ gate electrodes were then sputter-deposited for capacitance-voltage $(C-V)$ and currentvoltage $(I-V)$ measurements.

According to Kamiyama and co-workers, the actual wafer temperature during FLA is not known. XRD measurements on $\mathrm{HfO}_{2}$ films after rapid thermal annealing suggest that the crystallization temperature of $\mathrm{HfO}_{2}$ films is $\sim 600^{\circ} \mathrm{C}$. The onset of post-FLA crystallization for similar films begins at capacitor charging voltages of $3300 \mathrm{~V}$. $C-V$ and $I-V$ measurements on $\mathrm{HfO}_{2}$ gate dielectrics with $\mathrm{TaSi}_{2.5} / \mathrm{W}$ gate electrodes after xenon FLA show an increase in dielectric constant, and leakage current values comparable with similar samples after annealing in $\mathrm{O}_{3}$. Although the films show a slight decrease in equivalent oxide thickness $(\sim 5 \%)$ after FLA, the researchers said this process shows promise for manufacturing high- $\kappa$-based transistors with metal gates.

JEREMIAH T. ABIADE

\section{ZnO Field-Effect Transistors Fabricated on Self-Assembled Colloidal Nanorods}

Zinc oxide $(\mathrm{ZnO})$ is a transparent semiconductor with a large bandgap. $\mathrm{ZnO}$ thinfilm field-effect transistor (TFT) devices fabricated with colloidal $\mathrm{ZnO}$ nanocrystals are particularly attractive because they are based on a low-temperature, solutionprocessible semiconducting material. Researchers Baoquan Sun and Henning Sirringhaus of the University of Cambridge have now fabricated high-performance $\mathrm{ZnO}$ TFT devices on a $\mathrm{SiO}_{2} / \mathrm{Si}$ substrate by spin-coating an aqueous suspension of $\mathrm{ZnO}$ nanocrystals to form a thin film on the substrate.

As reported in the December 2005 issue of Nano Letters (DOI: 10.1021/nl051586w; p. 2408), the researchers investigated the effects of the nanocrystal shape on the colloidal self-assembly of the nanoparticles on the substrate and on the resulting device performance. They found that by changing the shape of the nanocrystals from sphere to rod, the semiconducting properties of the spin-coated $\mathrm{ZnO}$ films were greatly improved as the result of increased particle size and self-alignment along the substrate. Post-deposition hydrothermal growth of the nanorods in an aqueous zinc-ion solution was found to further enhance the nanorod dimensions and interconnectivity and significantly improve the device performance. TFT devices made from 65-nm-long, 10-nmwide nanorods exhibited a mobility of 0.61 $\mathrm{cm}^{2} \mathrm{~V}^{-1} \mathrm{~s}^{-1}$ and an on/off ratio of $3 \times 10^{5}$ after the post-deposition growth. These values are comparable to that of TFTs fabricated by traditional sputtering methods. The researchers also discovered that careful control of stoichiometry during the nanocrystal growth is crucial for achieving sufficiently low film conductivity and high field-effect mobility.

The researchers said that the use of nanorods instead of nanospheres as a seed layer for the hydrothermal growth resulted in long nanorods oriented preferentially in the plane of the substrate near the interface with the gate dielectric. This orientation is favorable for charge transport in TFTs. The researchers concluded, "making use of the self-assembly processes in colloidal nanocrystals is an attractive and simple route for controlling the microstructure and electronic properties of solution-processed semiconductor nanocrystal films."

SHIMING WU

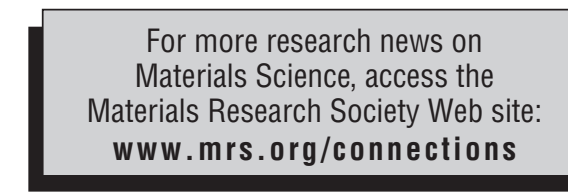

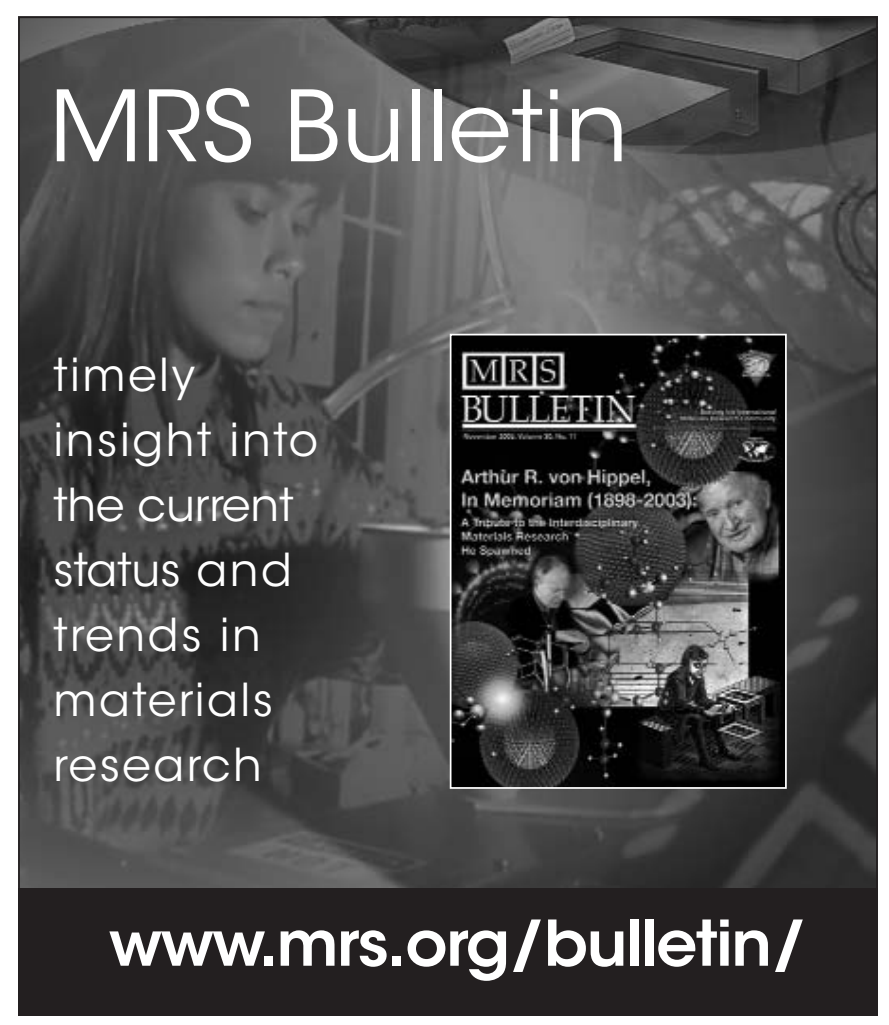

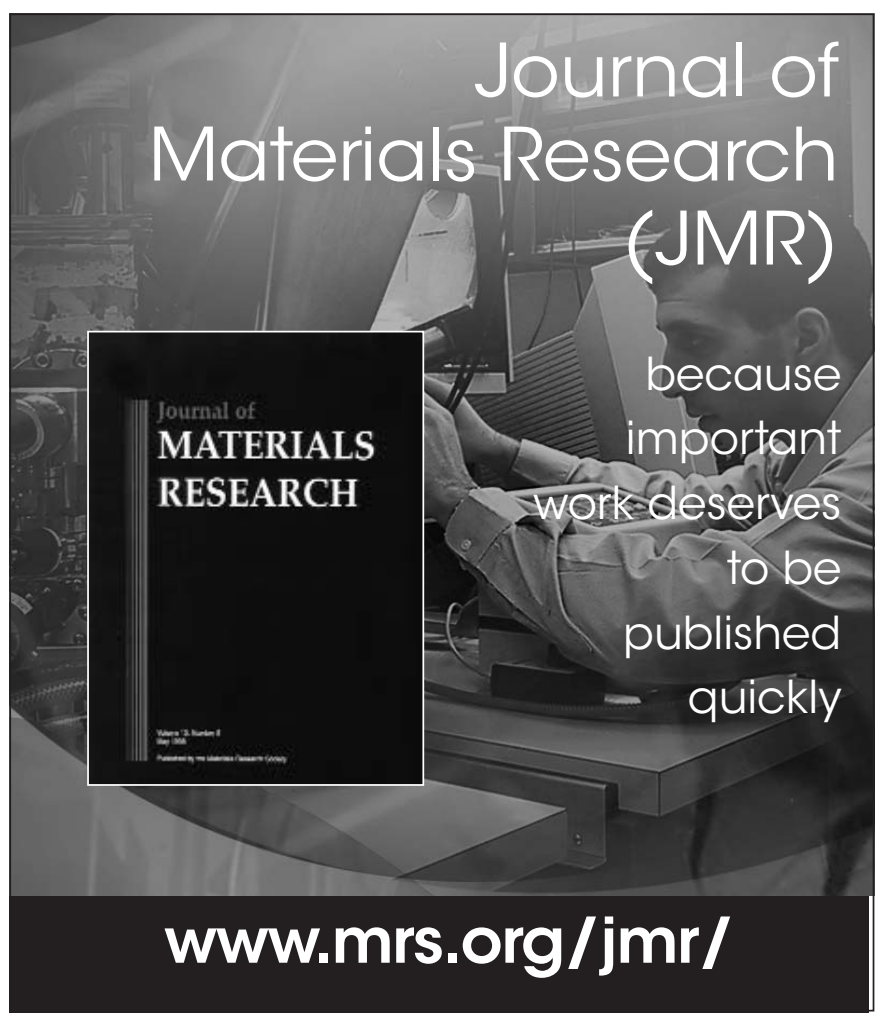

